\title{
Coexistence of low threshold lasing and strong coupling in microcavities
}

\author{
Pavlos G. Lagoudakis, ${ }^{\text {a) }}$ M. D. Martin, and Jeremy J. Baumberg \\ Department of Physics and Astronomy, University of Southampton, Southampton SO17 1BJ, \\ United Kingdom \\ Guillaume Malpuech and Alexey Kavokin \\ LASMEA, UMR-6602, Universit Blaise Pascal, 24 av. des Landais, 63177 Aubiere, France
}

(Received 30 April 2003; accepted 2 December 2003)

\begin{abstract}
We report the coexistence of low threshold lasing and strong coupling in a high-quality semiconductor microcavity under near-resonant optical pumping. A sharp laser mode splits from the lower-polariton branch and approaches the bare cavity mode frequency as the pump power increases. The lasing is produced by low density localized exciton states, which are weakly coupled to the cavity mode. The appearance of this lasing mode distinguishes quantum-well excitons into those which are strongly or weakly coupled with the cavity mode. () 2004 American Institute of Physics. [DOI: 10.1063/1.1643191]
\end{abstract}

A photon mode in a Fabry-Perot resonator and an excitonic resonance in a semiconductor quantum well $(\mathrm{QW})$ resemble two harmonic oscillators which can be either weakly or strongly coupled together in semiconductor microcavities containing embedded quantum wells. In the weak-coupling (WC) regime, resonant tuning of the cavity mode and the exciton transition results in formation of two degenerate states (a crossing behavior), while for strong-coupling (SC) on resonance, an energy splitting between new eigenstates of the system occurs (anticrossing situation). ${ }^{1} \mathrm{WC}$ exists in vertical cavity surface emitting lasers (VCSELs) and leads to enhancement of the spontaneous emission of excitons in resonance with the cavity mode. The SC regime has also been intensively studied in the past decade as it offers a unique opportunity to observe optical effects associated with exciton polaritons, i.e., quasiparticles composed of half a photon and half an exciton, coherently propagating in the microcavity plane. The transition between weak-/strongcoupling in microcavities has been carefully studied. ${ }^{2-5}$ This transition is governed by a condition on the exciton oscillator strength and decay rate, parameters that one can alter by changing the temperature, exciton density, or applying external fields. ${ }^{6}$

Here we report clear evidence of the simultaneous presence of the perturbative (weak) and nonperturbative (strong) regimes when optically exciting semiconductor microcavities. We observe an unambiguous anticrossing behavior of the coupled cavity and exciton modes, characteristic of the strong-coupling regime, while between these eigenstates of the system a new strong line appears whose intensity is exponentially dependent on the pumping power. An intermediate regime is identified where this lasing mode, which fulfills the characteristics of the weak-coupling regime, coexists with the free polariton modes. This extra mode results from the inversion of a population of localized exciton states. ${ }^{7}$ Population inversion at pump densities lower than the tran-

\footnotetext{
a) Author to whom correspondence should be addressed; electronic mail: p.lagoudakis@soton.ac.uk
}

sition threshold density is possible in microcavities possessing a very low concentration of localized states. Thus a VCSEL and strongly coupled exciton polaritons are simultaneously present in the same cavity.

The semiconductor structure used in this study is a conventional cavity with optical linewidth $\gamma_{c}=0.41 \mathrm{meV}$, and a single GaAs QW with exciton linewidth $\gamma_{x}=0.44 \mathrm{meV}$, fully discussed in Ref. 8 (studied under conditions that preclude charged exciton formation). The optical cavity length is $\sim \lambda$ and varies across the sample. A continuous-wave Ti:S pump laser at $1.557 \mathrm{eV}$ excites the QW above the band gap. The SC of the cavity mode with both heavy- and light-hole excitons $\left(X_{h h}\right.$ and $\left.X_{l h}\right)$ in the QW results in lower, middle, and upper polariton (LP), (MP), and (UP) branches (Fig. 1). In this way unbound electrons and holes are injected at energies $\sim 30 \mathrm{meV}$ above the heavy- or light-hole excitons in the structure. These carriers relax rapidly onto the lower polariton (LP) branch and create a reservoir of exciton polaritons at $1.5277 \mathrm{eV} ., 10$ This exciton-polariton population (which is termed the "exciton reservoir" due to the predominantly excitonic character of the high-wave vector exciton polaritons) is assumed to be proportional to the pumping power, $P$, and feeds the lower energy emitting states.

Photoluminescence (PL) spectra are taken at normal incidence $(\theta=0)$ within a cone of $\pm 0.15^{\circ}$ for a wide range of pump powers [Fig. 2(a)]. For pump powers above $200 \mu \mathrm{W}$, an extra mode appears, termed here the localized-exciton mode (LXM) for reasons explained below. The LXM emerges from the LP mode and shifts to higher energies with increasing pump power, saturating at the energy of the bare exciton mode. This dependence has been observed for a range of detuning $(\Delta)$ conditions of exciton and cavity mode (from $\Delta=+2$ to $-4 \mathrm{meV}$ ). At the same time, the peak intensity of the LXM versus pump power exhibits an almostexponential increase until saturating when its energy reaches the bare cavity mode. Surprisingly the polariton modes coexist with the LXM for a wide range of pump powers. However, for pump powers above $1 \mathrm{~mW}$ these modes clearly collapse and the system passes exclusively into the WC re- 


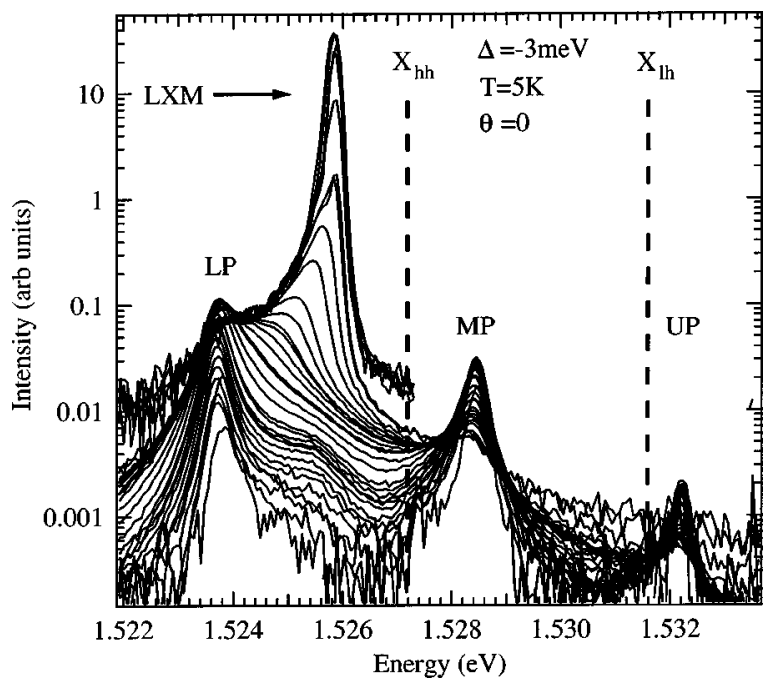

FIG. 1. Perpendicular PL emission for increasing (upwards) pumping intensities from $200 \mathrm{nW}$ to $6 \mathrm{~mW}$. The detuning, $\Delta=-3 \mathrm{meV}$.

gime. Further confirmation of the role of the cavity mode is seen in the detuning dependence [Fig. 3(a)] which again shows the coexistence of the anticrossing polaritons modes with the LXM, which follows the bare cavity mode.

To confirm this unexpected coexistence of strong- and weak-coupling regimes, we discount trivial explanations such as the coexistence of the two regimes due to the Gaussian excitation beam profile on the sample surface. The threshold in power ${ }^{11}$ would lead to an annulus of SC and a central spot of WC, changing in relative strength with increasing power. The inset to Fig. 2(a) shows the result of a calculation dividing the pumped area into such strong and weak-coupled domains, with the boundary between them radially increasing from the center of the pump spot as the power increases. We scale the calculation to match the experimentally observed appearance of the LXM, and incorporate the observed power dependence of lower polariton PL. The fact that the observed lower polariton PL does not decrease beyond the appearance of the LXM mode contrary to the above theory, together with the gradual energy shift of the LXM compared to its appearance directly at the bare cavity mode energy, shows that a simple collapse is not ob-

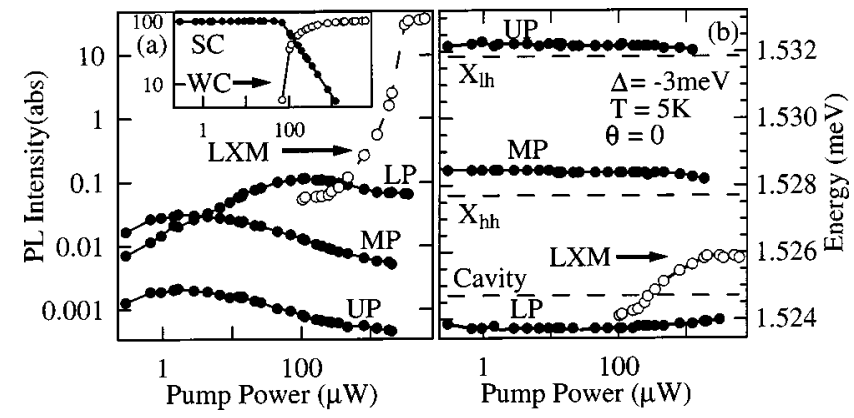

FIG. 2. (a) Normalized perpendicular PL emission vs $I_{\text {pump }}$, for polariton modes $(\mathbf{O})$ and the localized-exciton lasing mode $(\bigcirc)$. Inset: expected normalized PL for spatially inhomogeneous Rabi transition; (b) peak perpen-

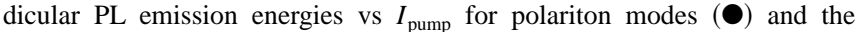
localized-exciton lasing mode $(\mathrm{O})$. The detuning, $\Delta=-3 \mathrm{meV}$.

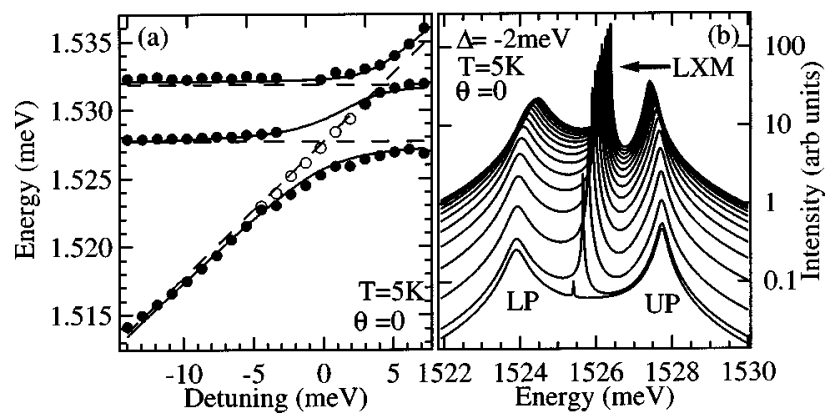

FIG. 3. (a) Experimental PL emission of upper, middle, and lower polariton modes $(\bigcirc)$ and the localized-exciton lasing mode $(O)$ together with theoretical dispersions of upper, middle, and lower polariton modes (solid lines), cavity, $X_{h h}$ and $X_{l h}$ modes (dashed lines), shown vs cavity detuning; (b) calculated emission from weakly and strongly coupled states. The pump evolves from $200 \mu \mathrm{W}$ to $3 \mathrm{~mW}$ in steps of $200 \mu \mathrm{W}$.

served. Another reason the LXM might be selected is from a resonance between excitation and emission energies when differing by a LO-phonon energy: this is excluded by the absence of any dependence on the excitation energy.

A basic interpretation for the appearance and evolution of this LXM can be derived starting from the coupled oscillator model. The strong coupling regime holds if $2 \Omega_{R}>\gamma_{x}$ $+\gamma_{c}$, where $\Omega_{R}$ is the exciton-cavity mode coupling (proportional to the square root of the exciton oscillator strength). In every semiconductor heterostructure both free and localized exciton states exist due to the disorder potential fluctuations. The latter states have a reduced oscillator strength and contribute to the inhomogeneous broadening of the exciton line out of resonance with the cavity mode (e.g., at large incident angles ${ }^{10}$ ), but do not contribute to microcavity PL spectra in the strong coupling regime, because emission and absorption of light are dominated by free polariton modes shifted far into the tail of localized states. ${ }^{12}$ Our results show that microcavities with extremely low concentration of localized states can be significantly populated by nonresonant optical excitation. This allows their population inversion, accompanied by low threshold lasing (absorbed pump power $P \simeq 0.4 \mathrm{~W} / \mathrm{cm}^{-2}$ ) once the gain is sufficient to offset round trip losses in the high finesse cavity. In this regime, the microcavity acts both as a VCSEL and a strongly coupled microcavity. This relies on the cavity mode being narrower than the inhomogeneous distribution of localized excitons (as is the case here). The situation is impossible in lower-quality cavities where the round trip losses are higher.

The lasing mode frequency increases with pump power $P$ because localized states at higher energy can now successively become inverted. This occupation of the QD distribution affects both the optical susceptibility and the emission of the microcavity. We assume that nonresonant pumping generates an exciton reservoir with an energy close to the free exciton energy, $\omega_{x}$. This reservoir has a population $N_{x}$ that is proportional to the external pumping power $P$. Relaxation from $N_{x}$ populates the inhomogeneous distribution (of width $\gamma_{x}$ ) of localized exciton states whose density $g(\omega)$ is taken to be Gaussian. We assume that at these low densities, collision between reservoir excitons is the main mechanism populating localized states whose total population $N_{\mathrm{LX}}$ is thus proportional to $P^{2}$. Acoustic phonon-exciton scattering 
is another mechanism for populating the localized exciton states. However, at the low temperature range of our study, inclusion of this mechanism does not qualitatively affect our results. We assume that localized states are sufficiently well thermalized to be described by a Boltzmann distribution defined by a chemical potential $\mu(P)$. All states having an energy smaller than $\mu$ are strongly populated, they cannot absorb light anymore and have therefore a vanishing oscillator strength. In the framework of the nonlocal semiclassical model, ${ }^{13}$ the QW susceptibility reads:

$$
\begin{aligned}
\tilde{\chi}(\omega, \gamma)= & \frac{1}{\pi^{1 / 2} \gamma_{x}} \int_{\mu}^{+\infty} \frac{\Gamma_{0}}{\omega_{x}-\omega-i \gamma} \\
& \times \exp \left[-\left(\frac{\omega_{1}-\omega_{x}}{\gamma_{x}}\right)^{2}\right] d \omega_{1},
\end{aligned}
$$

where $\Gamma_{0}$ is the radiative emission rate of the exciton, and $\gamma$ is its nonradiative homogeneous broadening. Once this susceptibility is known, one can use the generalized scattering state technique ${ }^{14}$ to calculate numerically the emission spectrum of light $F(\omega, \mu)$ escaping from the sample, if generated from a narrowband emitter placed in the quantum well. To obtain the spectral emission of the microcavity, $F(\omega, \mu)$ should be multiplied by the spectral distribution of the embedded emitters. We distinguish two main types of emitters. The first type are SC excitons which have an emission spectrum $I_{\mathrm{SC}}(\omega)$ proportional to the optical susceptibility, $(\tilde{\chi})$. The second type of emitters are inverted weakly coupled excitons. We assume that the main contribution from WC excitons comes from the states having an energy equal to the chemical potential. The intensity is therefore proportional to the number of localized states at the energy of the chemical potential. The spectral emission from weakly coupled excitons therefore reads (using for simplicity an arbitrary zero of energy at $\omega_{x}$ ):

$$
I_{\mathrm{LX}}(\omega, \mu)=\frac{1}{\pi^{1 / 2} \gamma_{x}} \frac{\Gamma_{0}}{\omega-\mu-i \gamma} \exp \left(-\mu^{2} / \gamma_{x}^{2}\right) .
$$

The total spectrum $I_{T}$ emitted by the sample is then

$$
I_{T}(\omega, \mu)=\left[I_{\mathrm{SC}}(\omega, \mu)+\alpha I_{\mathrm{LX}}(\omega, \mu)\right] F(\omega, \mu) .
$$

The only unknown parameters remaining are the phenomenological coefficient $\alpha$ which depends on the density of localized states produced by the disorder, and the power dependence of $\mu$. Solving

$$
N_{\mathrm{LX}}=\int g(\omega) \exp \left(\frac{\mu(P)-\omega}{k_{b} T}\right) d \omega,
$$

with Boltzmann's constant, $k_{b}$, and temperature, $T$, gives

$$
\mu(P)=\frac{\gamma_{x}^{2}}{2 k_{b} T} \ln \left(P / P_{c}\right) \text {. }
$$

When the critical pump power $P_{c}$ is reached, $\mu$ vanishes and the emission energy reaches the free exciton energy. Thus, $\mu$ represents the energy shift of the lasing with respect to the free exciton energy. Using the microcavity parameters and $P_{c}=3 \mathrm{~mW}$, the shift of the lasing mode as $P$ is increased to $P_{c}$ is $1 \mathrm{meV}$, in qualitative agreement with experimental findings.

The emission $I_{T}(\omega, P)$ is calculated in Fig. 3(b) with $\alpha=1$ and $\Delta=-2 \mathrm{meV}$ in order to allow direct comparison with Fig. 2(b). Many of the features in the experiment are reproduced by this highly simplified theory, including the shift in the laser emission $\propto \ln (P)$ and the exponential rise in LXM emission, supporting the identification of this lasing mode.

In summary, we show theoretically and experimentally that it is possible to have strongly coupled polariton modes and laser emission from localized exciton states in the same semiconductor microcavity. In high quality microcavities, the small density of low energy localized states weakly coupled to the cavity mode can be inverted, and provide sufficient gain to overcome cavity mode losses. This work thus links the fields of VCSELs and strongly coupled microcavities, important for future generations of highly efficient semiconductor emitters.

The authors are grateful for critical comments from R. Zimmermann and acknowledge the support of the HEFCE JR98SOBA, EC CLERMONT HPRN-CT-1999-00132, and EPSRC GR/N18598.

${ }^{1}$ C. Weisbuch, M. Nishioka, A. Ishikawa, and Y. Arakawa, Phys. Rev. Lett. 69, 3314 (1992).

${ }^{2}$ R. Houdré, J. L. Gibernon, P. Pellandini, R. P. Stanley, U. Oesterle, C. Weisbuch, J. O'Gorman, B. Roycroft, and M. Ilegems, Phys. Rev. B 52, 7810 (1995).

${ }^{3}$ R. Butté, G. Delalleau, A. I. Tartakovskii, M. S. Skolnick, V. N. Astratov, J. J. Baumberg, G. Malpuech, A. Di Carlo, A. V. Kavokin, and J. S. Roberts, Phys. Rev. B 65, 205310 (2002).

${ }^{4}$ R. P. Stanley, R. Houdré, C. Weisbuch, U. Oesterle, and M. Ilegems, Phys. Rev. B 53, 1099511007 (1996).

${ }^{5}$ M. Emam-Ismail, V. N. Astratov, M. S. Skolnick, D. M. Whittaker, and J. S. Roberts, Phys. Rev. B 62, 1552 (2000).

${ }^{6}$ M. S. Skolnick, T. A. Fisher, and D. M. Whittaker, Semicond. Sci. Technol. 13, 645 (1998).

${ }^{7}$ X. Fan, H. Wang, H. Q. Hou, and B. E. Hammons, Phys. Rev. B 56, 15256 (1997).

${ }^{8}$ R. Rapaport, E. Cohen, A. Ron, E. Linder, and L. N. Pfeiffer, Phys. Rev. B 63, 235310 (2001).

${ }^{9}$ F. Tassone, C. Piermarocchi, V. Savona, and P. Schwendimann, Phys. Rev. B 56, 7554 (1997).

${ }^{10}$ R. Huang, Y. Yamamoto, R. André, J. Bleuse, M. Muller, and H. UlmerTuffigo, Phys. Rev. B 65, 165314 (2002).

${ }^{11}$ G. Khitrova, H. M. Gibbs, F. Jahnke, M. Kira, and S. W. Koch, Rev. Mod. Phys. 71, 1591 (1999).

${ }^{12}$ A. V. Kavokin, Phys. Rev. B 50, 8000 (1994).

${ }^{13}$ L. C. Andreani, G. Panzarini, A. V. Kavokin, and M. R. Vladimirova, Phys. Rev. B 57, 4670 (1998).

${ }^{14}$ G. Malpuech and A. Kavokin, Semicond. Sci. Technol. Top. Rev. 16, R1 (2001) 